\title{
Depression Anxiety Outcome: Insomnia and Up-to-date
}

\section{Treatments}

\author{
Habib A. Rabbani \\ Department of Psychology, Education and Human Sciences, Universities of Tabriz and Tehran (Roodehen), Iran
}

\begin{abstract}
Based on his teaching experiences and clinical observations at Mental Health Centers, the author presents shortly the results of his clinical researches and will discuss on "psychopathological effects of depression and anxiety on insomnia" and very summarily on the treatment. The studies and lectures at the University Hospitals: Ir., IL, USA and France, especially during the last decades, have enriched this study research. The experiences and researches have clearly shown that depression and anxiety resulting from catastrophic life events, exert pathological effects not only on cognitive and cardiovascular system but have also psychopathological effects on internal organs such as pineal gland with its melatonin secretion which is considered as stimulator system to regulate the rhythm of wake-sleep. Major depression and anxiety, when accumulating and occurring together, can not only perturb the circadian rhythm, which leads to insomnia, but also in some extreme situations as observed, can engender behavior and personality disorders that in some cases lead to suicide. Concerning the treatments in some cases in which the parents in major depression are suffering from the death of children, in parallel of pharmacotherapy, or other family care and treatments, the author proposed "Child Replacement” plan in period of mourning. Among the various drugs effective for treatment of insomnia we indicate: Agomelatine-Mianserine-Amitriptyline-Doxepin-Fluoxetine, well interesting to be noted.
\end{abstract}

Key words: Depression, anxiety, insomnia, psychotherapy, melatonin.

\section{Introduction}

In this research paper, based on his teaching experiences and clinical observations in Mental Health Centers, the author will discuss briefly on the psychopathological effects of depression and anxiety on insomnia and/or headache; then very summarily on their treatment.

Resulting from anxiety, depression and in some cases psychosomatic disorders, or from life's inconveniences, insomnia in our time is very common and became a significant source of major complaints and underdiagnosed. It is to add here that insomnia is not new phenomenon, it has existed throughout history and traditional medicine, but the etiology and physiopathology were not clear; the treatment, without any real definition or analysis of their biological or psychosocial factors and reasons, has been generally on

Corresponding author: Habib A. Rabbani, Ph.D., Ex Prof., researcher (Paris, France), research fields: clinical psychology, psychogeriatry, post-traumatic stress disorder (PTSD). the basis of medical plants and traditional or local methods as we observed also into the television; so, clinical studies in mental disorders have been very limited or confined to some modest hospitals and environmental factors, also quality of life etc. of the patients were neglected. Since World War 2, as the author can remember, particularly during the 4th past decade and in our present time, because of psychosocial and political changes: immigration (especially forced one [1] as observed in many cases); catastrophic life events: human and nature, etc., depression, anxiety or many other mental and physical disasters have been recognized. As our experiences and clinical studies have clearly indicated, any pathological process or disturbance in brain and body may have an harmful influence not only on the cognitive system [2] and cardiovascular system [3], but also other systems and organs such as internal glands, discussed elsewhere [4] and consequently leading to insomnia, headache etc.

These multifactorial reasons (Fig. 1), mentioned 
above, specially war and violence, with their catastrophic consequences [5], acts of terrorism and other factors presented elsewhere are the prominent reasons that can affect the quality of life and induce psychosomatical and mental problems: depression, anxiety etc. and as the author has noted, in many cases finally leading to headache and/or insomnia.

Concerning treatments, in all cases (chronic, acute or other forms of insomnia) as noted, the correct diagnosis (blood analysis, scanner, electroencephalogram (EEG) etc.) must be performed beforehand. Our experiences and clinical studies have shown the combination of two principal methods: psychotherapy and pharmacological therapy both are necessary. During the consultations or clinical interviews, the description of patient suffering from insomnia is primordial. In many cases, as observed, when the insomnia resulted from depression, anxiety or other catastrophic factors mentioned above, it is necessary to take beforehand some antidepressants (ex. agomelatine, amitriptylin, doxepin, nortriptyline) or tranquilizers ...discussed in Ref. [6] that, in spite of their side effects (dry mouth, constipation, sweating etc.) can reduce relatively the depression, then find drugs dedicated to the treatments of insomnia (ex. melatonin [7]). However, in spite of the efficacy of this hormone to improve the quality of sleep, our clinical experiences have clearly shown that it is convenient to maximise the up-to-date dosage.

\section{Discussion}

Our experiences have shown that in parallel of psychotherapy for insomnia, the use of regular sports and physical activities, as discussed in Ref. [8] or some positive occupations: music [9], television ...can play a role to reduce and limit depression and anxiety, thus may gradually improve the insomnia. Moreover, a complete hygiene will be important to reduce relatively the insomnia.

In some situation or cases (major depression), for example in which the parents are suffering from the loss or death of children in the period of mourning time, as noted in Fig. 1, we have proposed, in parallel of pharmacotherapy for the family, a "Child Replacement”; in two cases, as we observed, the efficiency of this complementary method was relatively well.

\section{Conclusion}

Considering the teaching experiences, clinical observations at Mental Health Centres of Hospital Universities in one side and also theoretical studies during last decades in Paris, Saint Anne, Lariboisière and La Salpêtriere, as mentioned above, in the other side, the author made a very short introduction about insomnia. Some factors triggering the insomnia noted in Fig. 1 have been presented; we understood clearly that depression and anxiety, resulting from the multi-factors noted above, are two principal contributors for Insomnia. We do not deny noting that in some cases major depression and anxiety, when accumulating and occurring (or coexisting) together, as also studied by Fava [10] can perturb the melatonin secretion (Fig. 2), and so the circadian rhythm system and finally lead to insomnia.

\section{Acknowledgements}

Many thanks to:

(1) Prof. H. Loo and Prof. J. P. Olié for their Seminars of Psychiatry, organized in Sainte Anne University Hospital from 1988 to 2008;

(2) Prof. M. Haguenau, Prof. C. Lidy and colleagues 1996-1998 for their teaching concerning "Cerebral Ageing Normal and Pathological" at the Faculty of Medicine Lariboisiere (Saint-Louis) University of Paris VII;

(3) Prof. Ph. Mazet, director of teachings, University of Paris VI, Dr. J. M. Thurin, professor and director of teaching at Faculty of Medicine, University of Pierre et Marie Curie, 


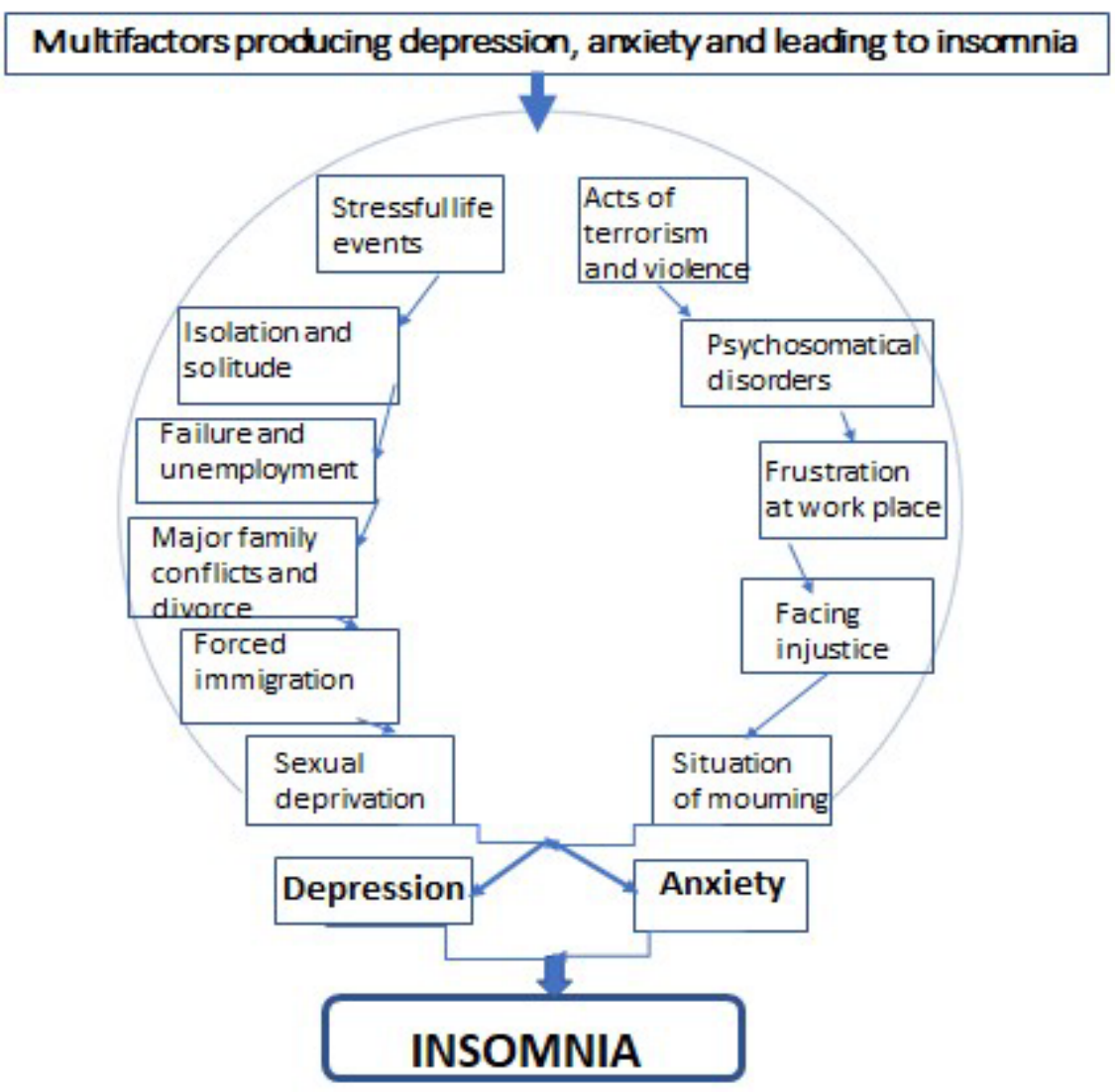

Fig. 1 Factors producing depression, anxiety, leading to insomnia.

\section{Pineal gland}

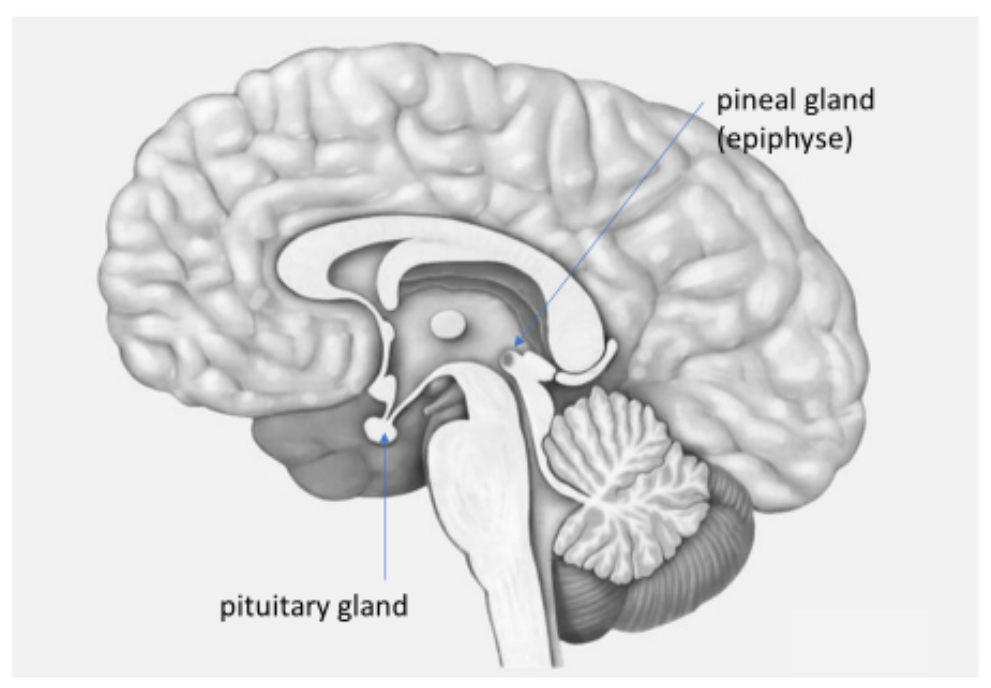

Fig. 2 Pineal gland secreting melatonin.

to obtain diploma on: "STRESS, TRAUMATISM $\quad$ (4) Dr. N. Bendersky for her kind help. AND PATHOLOGY”; 


\section{References}

[1] Rabbani, H. 1991. "Personality Disorders in Older Adults and Ageing after Immigration.” Medical Psychology 23 (3): 303-7.

[2] Rabbani, H. 2000. "The Effects of Traumatic Stress and Depression on Behaviour Disorders and Cognitive System.” Presented at International Congress of Scientific Psychology, Stockholm, Sweden.

[3] Rabbani, H. 2016. "Psychopathological Effects of Post Traumatic Stress Disorder on Brain and Heart.” Presented at the 3rd International Conference on Heart and Brain, Paris.

[4] Rabbani, H. 2018. "Sleep Disorders in Older Adults and Elderly Following the Catastrophic Stressful Life Events." Journal of Health Science 6 (5): 351-4.

[5] Rabbani, H. 2003. "Pourquoi la guerre et la violence, et non pas la paix et la tolérance.” Presented at 21st World Congress of Philosophy, Istanbul, Turquie.

[6] Rabbani, H. 2008. "Pharmacotherapy and Psychosocial
Care in the Treatment of Depression in Ageing.” Presented at XXVI International Congress CINP, Munich.

[7] Wade, A. G., Laudon, M., et al. 2007. "Efficacy of Prolonged Release Melatonin in Insomnia Patients Aged 55-80 Years: Quality of Sleep and Next-Day Alertness Outcomes.” Current Medical Research and Opinion 23 (10): 2597-605.

[8] Rabbani, H. 2007. "Psychosocial Effects of Sport and Physical and Mental Activities in Change and Reduction of Anxiety and Depression.” $12^{\text {th }}$ European Congress of Sport Psychology, Halkidiki, Greece.

[9] Rabbani, H. 1992. "Musique et Musicothérapie comme un traitement.” Congrès international des Arts et Psychiatrie, Faculté de Médecine de Tours, Tours, France.

[10] Fava, M. 2008. "Addressing Comorbidities in Insomnia-Advancing Insomnia Therapy.” XXVI Congress of the Collegium Internationale Neuro-Psychopharmachologicum (CINP), Munich, Germany. 